\title{
First-Principles Study of the Structural Stability and Electronic and Elastic Properties of Helium in $\alpha$-Zirconium
}

\author{
Jian Zheng, ${ }^{1,2}$ Huijun Zhang, ${ }^{2,3}$ Xiaosong Zhou, ${ }^{2}$ Jianhua Liang, \\ Liusi Sheng, ${ }^{1}$ and Shuming Peng ${ }^{2}$ \\ ${ }^{1}$ School of Nuclear Science and Technology, University of Science and Technology of China, Hefei 230026, China \\ ${ }^{2}$ Institute of Nuclear Physics and Chemistry, China Academy of Engineering Physics, Mianyang 621900, China \\ ${ }^{3}$ School of Mathematics and Physics, University of Science and Technology Beijing, Beijing 100083, China \\ Correspondence should be addressed to Shuming Peng; pengshuming@hotmail.com
}

Received 22 January 2014; Revised 11 April 2014; Accepted 20 April 2014; Published 5 May 2014

Academic Editor: Haiyan Xiao

Copyright ( 2014 Jian Zheng et al. This is an open access article distributed under the Creative Commons Attribution License, which permits unrestricted use, distribution, and reproduction in any medium, provided the original work is properly cited.

\begin{abstract}
First-principles calculations within density functional theory have been performed to investigate the behaviors of helium in $\alpha$ zirconium. The most favorable interstitial site for $\mathrm{He}$ in $\alpha-\mathrm{Zr}$ is not an ordinary tetrahedral or octahedral site, but a basal octahedral site with a formation energy as low as $2.40 \mathrm{eV}$. The formation energy reduces to $1.25 \mathrm{eV}$ in the presence of preexisting vacancies. The analysis on the density of states and the charge density has been carried out. In addition, the influences of $\mathrm{He}$ and small $\mathrm{He}-\mathrm{V}$ complexes on the elastic properties have been studied. The He-V complexes have been found to greatly affect the elastic properties compared with $\mathrm{He}$ alone.
\end{abstract}

\section{Introduction}

During service lifetime of materials used in nuclear energy systems, the impact of helium has been considered as a critical issue. Helium is almost insoluble in metals due to its inert reactivity with other elements. Once introduced, it can hardly be outgassed and will have profound deleterious effects on the microstructures and mechanical properties. Helium has been a topic of sustained interest during the past several decades for both nuclear material engineering and fundamental research. In particular, numerous experimental and theoretical studies have been dedicated to addressing the properties of He in metals such as $\mathrm{Ti}[1,2], \mathrm{Fe}[3,4]$, and $\mathrm{W}[5,6]$. It is known to affect the nucleation and growth of voids in metals, causing noticeable dimensional changes and significant hardening [7-10].

Zirconium and its alloys are widely used in tritium storage [11-13] and nuclear fission energy systems [14-18] owing to their high tritium storage density, low thermal neutron absorption cross-section, good aqueous corrosion resistance, and favorable mechanical properties. And they are believed to have some potential uses in future fusion applications
[19]. Under these application conditions, helium can be introduced by three principal ways: tritium $\beta$ decay, $(\mathrm{n}, \alpha)$ reactions, and direct He-ion implantation [20]. The tritium stored in zirconium can decay to helium by $\beta$ decay in the following nuclear equation: ${ }_{1}^{3} \mathrm{~T} \rightarrow{ }_{2}^{3} \mathrm{He}^{+}+\mathrm{e}^{-}+\bar{v}_{e}$. In fission reactors, because of the Ni doped in zirconium alloy and the $\mathrm{B}$ doped in the primary-loop water, the neutron flux may cause generation of helium either by (a) the two-step reaction with ${ }^{58} \mathrm{Ni}(\mathrm{n}, \gamma)$ and ${ }^{59} \mathrm{Ni}(\mathrm{n}, \gamma)$ or by (b) the ${ }^{10} \mathrm{~B}+\mathrm{n} \rightarrow{ }^{7} \mathrm{Li}+\alpha$ reaction [21]. In fusion reactors, $\mathrm{He}^{++}$and $\mathrm{He}^{+}$ions produced in the plasma can directly implant into materials.

However, as far as we know, only a few studies have been centered on the influences of $\mathrm{He}$ on $\mathrm{Zr}$, mainly some experimental works on the surface phenomenology [22, 23] and $\mathrm{He}$ bubble formation $[12,24]$. It is found that helium can easily form bubbles and these bubbles preferentially distribute in grain boundaries and dislocations. For direct He-ion implantation, blisters, even exfoliations, are evident at a critical dose [22, 23].

The pioneering theoretical study by Koroteev et al. [25] gives us a better understanding on the structure stability and electronic properties of the $\mathrm{Zr}-\mathrm{He}$ system. However, 
TABLE 1: Experimental and calculated lattice constants $(\AA)$ for $\alpha$-Zr.

\begin{tabular}{lcc}
\hline & $a$ & $c / a$ \\
\hline This work & 3.231 & 1.600 \\
PW91/PAW [30] & 3.213 & 1.605 \\
PW91/UPP [31] & 3.23 & 1.604 \\
PW91/UPP [32] & 3.223 & 1.606 \\
Exp. [33] & 3.23 & 1.593 \\
\hline
\end{tabular}

their calculations were performed in a rather small system containing only two $\mathrm{Zr}$ atoms and one $\mathrm{He}$ atom, which corresponds to an extremely high helium concentration. As we know, the stability of self-interstitial atom in $\alpha$ - $\mathrm{Zr}$ is strongly affected by the system size [26-29]. So, it is necessary to further their studies concerning a larger system size.

In the present paper, through first-principles calculations we reported helium's behavior in $\alpha$-Zr by focusing on the relative stabilities of single He defects, the electronic properties of $\mathrm{He}$ atom, and its nearest-neighbor $\mathrm{Zr}(\mathrm{NN}-\mathrm{Zr})$ atom. We also studied the changes of elastic properties due to the introduction of He atoms and vacancies.

\section{Computational Method}

The present calculations were carried out using CASTEP [39]. Ultrasoft pseudo potential (USPP) [40] with a consistent cutoff energy of $450 \mathrm{eV}$ based on density functional theory (DFT) was used. The Perdew, Burke, and Ernzerhof (PBE) form [41] of generalized gradient approximation (GGA) was applied as the exchange-correlation potential since it has been suggested by Domain et al. [31] that GGA performs better than the local density approximation (LDA) to describe the exchange-correlation functional of $\mathrm{Zr}$.

During the relaxations, the Brillouin zone (BZ) integration was achieved using a cold smearing scheme (MethfesselPaxton), with a smearing of sigma $=0.1 \mathrm{eV}$. BZ sampling was performed using the Monkhorst-Pack scheme, with a k-point spacing as close as possible to $0.030 \AA^{-1}$ (i.e., $4 \times 4 \times 3 \mathrm{k}$-point meshes for a 36 -atom supercell and $4 \times 4 \times 2 \mathrm{k}$-point meshes for a 54-atom supercell). The supercells were optimized using the Broyden-Fletcher-Goldfarb-Shanno (BFGS) [42] algorithm allowing both atomic positions and the lattice parameters to relax. The energy convergence criterion for self-consistent calculations was set as $1 \times 10^{-6} \mathrm{eV}$. The criteria for ionic energy minimization were set as energy derivative $<1 \times 10^{-5} \mathrm{eV} /$ atom, forces on individual atoms $<0.01 \mathrm{eV} \AA^{-1}$, displacement of atoms derivative $<0.001 \AA$, and stress component on cell $<0.05 \mathrm{GPa}$. The criteria for the calculation of elastic constants were set as energy derivative $<2 \times$ $10^{-6} \mathrm{eV} /$ atom, forces on individual atoms $<0.006 \mathrm{eV} \AA^{-1}$, and displacement of atoms derivative $<2 \times 10^{-4} \AA$. The parameters selected above ensure the convergence of formation energies within $0.001 \mathrm{eV} /$ atom. Spin polarization is not considered in all the calculations for the sake of time; our future work will involve it.

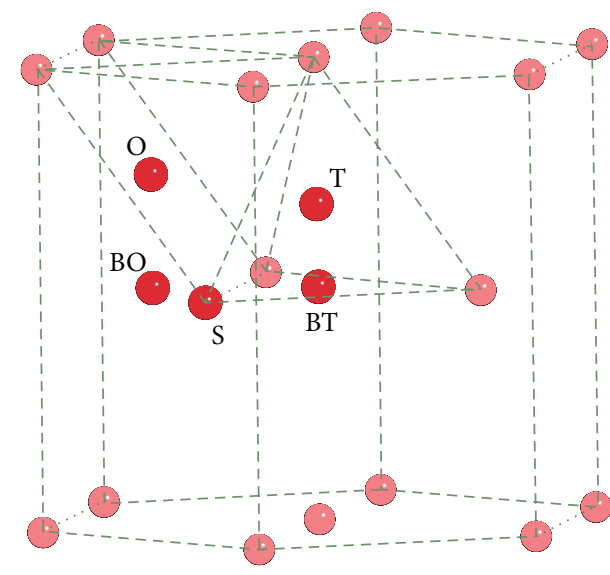

FIGURE 1: Five crystallographically unique interstitial sites in $\alpha$ zirconium (hcp structure), where the pink spheres are the hcp lattice sites and the red ones are labeled as octahedral $(\mathrm{O})$, basal octahedral (BO), tetrahedral (T), basal tetrahedral (BT), and substitutional (S) sites, respectively.

The defect formation energy is defined as

$$
E_{\text {defect }}^{f}=E_{m Z \mathrm{Zr}, \mathrm{He}}-m E_{\mathrm{Zr}}-E_{\mathrm{He}} \text {, }
$$

where $E_{m \mathrm{Zr}, \mathrm{He}}$ is the total energy of an optimized supercell containing $m \mathrm{Zr}$ atoms and one $\mathrm{He}$ atom, $E_{\mathrm{Zr}}$ is the energy per $\mathrm{Zr}$ atom in optimized crystal, and $E_{\mathrm{He}}$ is the energy of an isolated $\mathrm{He}$ atom.

\section{Computational Results}

3.1. Structural Stability. We first investigate the lattice constants of an $\alpha-\mathrm{Zr}$ crystal and the results are summarized in Table 1, compared with experimental and other calculated results. It can be seen clearly that our calculated results are in good agreement with the corresponding experimental and other calculated values.

When $\mathrm{He}$ atoms are introduced into a metal, they may occupy either substitutional or interstitial lattice sites. It is necessary to figure out the locations of $\mathrm{He}$ atoms since they will influence the solubility and migration. It is also important for the understanding of such effects as damage trapping, bubble nucleation, embrittlement, and blistering [6]. It has been found that, in different metals, He atoms preferentially occupy different sites: substitutional sites for $\mathrm{Fe}, \mathrm{Cr}, \mathrm{Mo}$, and W [6, 43], tetrahedral sites for Er [34], and FC (the center of equilateral trigonal face shared by two adjacent octahedrons) sites for Ti [2]. To identify the lowest energy configurations of the He interstitial defects in $\mathrm{Zr}$ and the possible influences of He concentration, supercells with 35 to $54 \mathrm{Zr}$ atoms and one $\mathrm{He}$ atom were studied. Five crystallographically unique interstitial sites for $\mathrm{He}$ atom were taken into consideration (see Figure 1). They were denoted conventionally as octahedral $(\mathrm{O})$, basal octahedral $(\mathrm{BO})$, tetrahedral (T), basal tetrahedral (BT), and substitutional (S) sites.

The calculated formation energies of a single vacancy and $\mathrm{He}$ atom in above sites are listed in Table 2, together 
TABLE 2: Vacancy formation energies (in $\mathrm{eV}$ ) for a single $\mathrm{He}$ atom positioned in different sites. All the configurations are found to be stable, except $\mathrm{O}$, which is unstable and transfers to BO. $E_{\mathrm{v}}^{f}$ represents the formation energy of a $\mathrm{Zr}$ vacancy and $E_{\mathrm{s}}^{\prime f}$ represents the formation energy of a substitutional $\mathrm{He}$ atom in a preexisting $\mathrm{Zr}$ vacancy.

\begin{tabular}{lccccccc}
\hline & $E_{\mathrm{v}}^{f}$ & $E_{\mathrm{s}}^{f}$ & $E_{\mathrm{s}}^{\prime f}$ & $E_{\mathrm{T}}^{f}$ & $E_{\mathrm{O}}^{f}$ & $E_{\mathrm{BT}}^{f}$ & $E_{\mathrm{BO}}^{f}$ \\
\hline 36-atom cell & 1.97 & 3.30 & 1.33 & 2.67 & $/$ & 3.01 & 2.42 \\
54-atom cell & 1.95 & 3.20 & 1.25 & 2.64 & $/$ & 2.99 & 2.40 \\
$\alpha$-Zr [25] & - & - & - & 3.08 & 3.19 & - & - \\
$\operatorname{Er}[34]$ & - & 2.59 & - & 1.73 & 1.98 & - & - \\
$\mathrm{Sc}[35]$ & - & 2.98 & - & 1.74 & 2.06 & 1.76 & 1.97 \\
$\operatorname{Ti}[2]$ & - & 3.65 & 1.70 & 2.82 & 3.04 & - & 2.69 \\
\hline
\end{tabular}

with the results reported in the literature for $\mathrm{Zr}$ and some other hcp-structure metals. It is noticeable that, in both supercells, the configuration $\mathrm{O}$ is not stable and decays to configuration BO during relaxation. Different from those of $\mathrm{Fe}, \mathrm{Cr}, \mathrm{Mo}$, and $\mathrm{W}$, the favorable sites in energy for $\mathrm{He}$ atom in $\alpha-\mathrm{Zr}$ are interstitial sites. Among the three stable interstitial sites, the most favorable one is the $\mathrm{BO}$ site in both supercells, with formation energy near $2.40 \mathrm{eV}$. However, it should be mentioned that $E_{\mathrm{s}}^{f}$ here actually represents the energy required to form a He-V complex. The formation energy of $1.33 \mathrm{eV}$ for a $\mathrm{He}$ atom in a preexisting vacancy (denoted as $E_{\mathrm{s}}^{\prime f}$ ) can be obtained by subtracting the formation energy of the vacancy (denoted as $E_{\mathrm{v}}^{f}$ ). It is much smaller than $E_{\mathrm{BO}}^{f}$, which means that He atoms can be trapped easily by preexisting vacancies. This is consistent with the case of $\mathrm{Ti}$ [2]. For materials used in nuclear systems, energetic particles like neutrons, ions, or electrons can induce significant microstructure alteration, especially the generation of large concentrations of vacancies, which can strongly affect the $\mathrm{He}$ distribution. In general, the stabilities of these configurations, in descending order, are preexisting vacancy, BO, T, BT, S, and $\mathrm{O}$. Koroteev et al. [25] also reported that the configuration $\mathrm{T}$ is more stable than configuration O. However, they did not take other configurations into consideration. And maybe due to the differences in calculating methods or system sizes, their calculated configuration $\mathrm{O}$ does not decay to BO. This is left to be clarified by further studies.

3.2. Electronic Properties. To further understand the relative stability of these structures with $\mathrm{He}$ at various sites, we also studied the DOS of the He atoms and the NN-Zr atoms of different configurations. The relative stability of the $\mathrm{He}$ at $\mathrm{BO}$ site to the other sites can be easily understood according to the position of the Fermi level relative to the peaks in the DOS, which determines the occupation of the states and the nature of bonding [44]. As shown in Figure 2(a), the interaction of basal octahedral He interstitial with its $\mathrm{NN}-\mathrm{Zr}$ atoms leads to the lowest DOS at the Fermi level which indicates that there is a stronger bonding between $\mathrm{Zr}$ atom and $\mathrm{He}$ atom at the $\mathrm{BO}$ site compared with other sites. This is in accordance with the formation energy differences as mentioned above. Different from those for Fe [43], it is also noticeable that the interaction also leads to different changes of the DOS at the Fermi level of the NN-Zr atoms, as shown in Figure 2(b). Comparing with that of the T and BT interstitial sites, the BO sites occupy a lower DOS at the Fermi level. The DOS at the Fermi level of the S site is somewhat particular because of the lack of one $\mathrm{Zr}$ atom. From Figure 3, we observe similar DOS peaks of the He-s and Zr- $d$ states at around $-1 \mathrm{eV}$ and Fermi level, which suggests the strong hybridization between these states. And this hybridization between $\mathrm{He}$ and metals has also been confirmed by other researchers $[34,35,43]$. It is believed that the DOS peaks of the He-s and M- (metals-, like Zr, Sc, Er, Fe, etc.) $d$ states will change due to the strong hybridization. This change will lead to an overall similarity in the shapes of those DOS curves.

We also calculated the total charge density $(\rho(r))$ of pure $\alpha$-Zr crystal and one with preexisting vacancies. The isosurfaces of $\rho(r)=140 \mathrm{e} / \mathrm{nm}^{3}$ and the contour plots of the charge density of the basal planes crossing the $\mathrm{BO}$ and $\mathrm{S}$ sites are illustrated in Figures 4(a) and 4(b), respectively. The isosurfaces are tubes similar to those of $\mathrm{Ti}$ [2]. Since the charge density inside the isosurfaces is lower than that of the outside, He atoms prefer to stay inside, especially in the BO or $\mathrm{S}$ sites, over which the tubes cross with the basal planes. It is obvious that the space inside the tube near the vacancy is much larger than that of the $\mathrm{BO}$ interstitial site. And this may explain why $\mathrm{He}$ atoms prefer to stay in preexisting vacancies.

3.3. Elastic Properties. Then, we studied the influences of helium on the elastic constants of Zr. For hexagonal structure materials, there are six elastic stiffness constants; five of them are independent $\left(C_{11}, C_{12}, C_{13}, C_{33}\right.$, and $\left.C_{44}\right)$ with $C_{66}=$ $\left(C_{11}-C_{12}\right) / 2$. The elastic constants were determined from the second derivatives of the total energy with respect to suitable deformations. The bulk modulus $B$ and shear modulus $G$ of the polycrystal were evaluated from the elastic constants of a single crystal by employing the Voigt-Reuss-Hill method [45-47]. For hexagonal lattices, the Reuss and Voigt bulk modulus $\left(B_{\mathrm{R}}\right.$ and $\left.B_{\mathrm{V}}\right)$ and the Reuss and Voigt shear $\left(G_{\mathrm{R}}\right.$ and $\left.G_{V}\right)$ can be defined as [45-47]

$$
\begin{gathered}
B_{\mathrm{R}}=\frac{C_{33}\left(C_{11}+C_{12}\right)-2 C_{13}^{2}}{C_{11}+C_{12}+2 C_{33}-4 C_{13}}, \\
B_{\mathrm{V}}=\frac{1}{9}\left[2\left(C_{11}+C_{12}\right)+C_{33}+4 C_{13}\right], \\
G_{\mathrm{R}}=\frac{5}{2} \frac{C_{44} C_{66}\left[C_{33}\left(C_{11}+C_{12}\right)-2 C_{13}^{2}\right]}{3 B_{V} C_{44} C_{66}+\left(C_{44}+C_{66}\right)\left[C_{33}\left(C_{11}+C_{12}\right)-2 C_{13}^{2}\right]}, \\
G_{\mathrm{V}}=\frac{1}{30}\left(C_{11}+C_{12}+2 C_{33}-4 C_{13}+12 C_{44}+12 C_{66}\right), \\
G=\frac{1}{2}\left(G_{R}+G_{V}\right), \\
B=\frac{1}{2}\left(B_{R}+B_{V}\right) .
\end{gathered}
$$




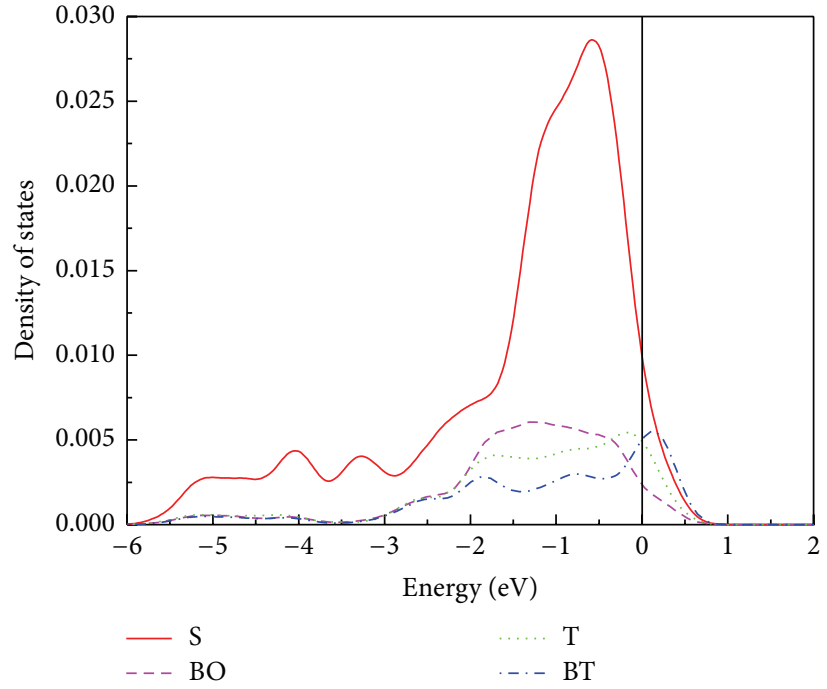

(a)

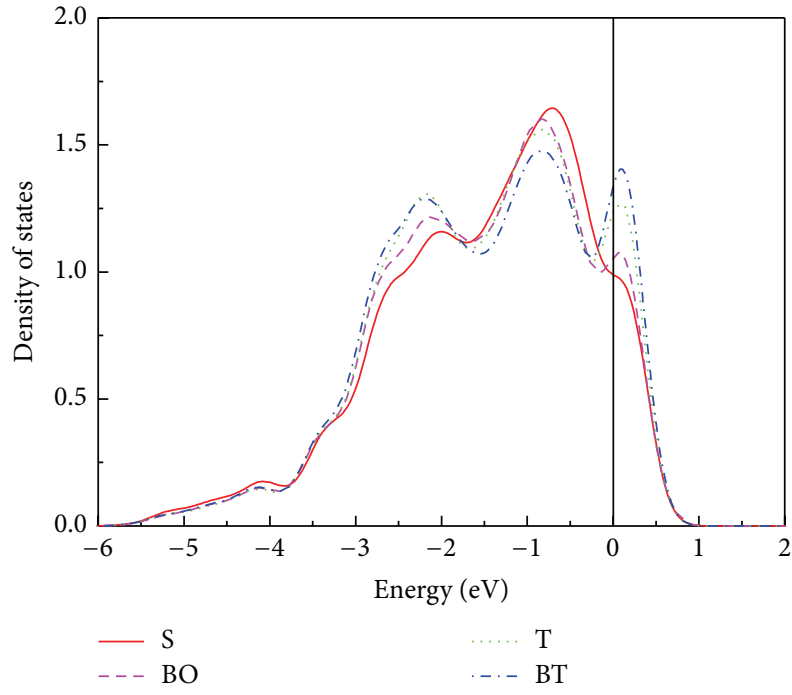

(b)

Figure 2: Total DOS of (a) an interstitial He atom at various positions and (b) its NN-Zr atoms (vertical line is the Fermi level $E_{F}$ ).

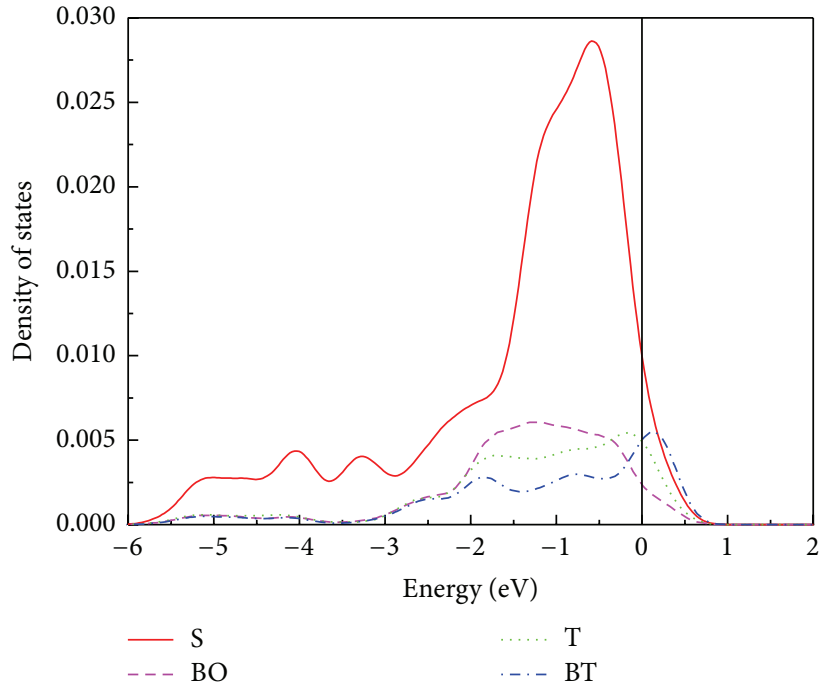

(a)

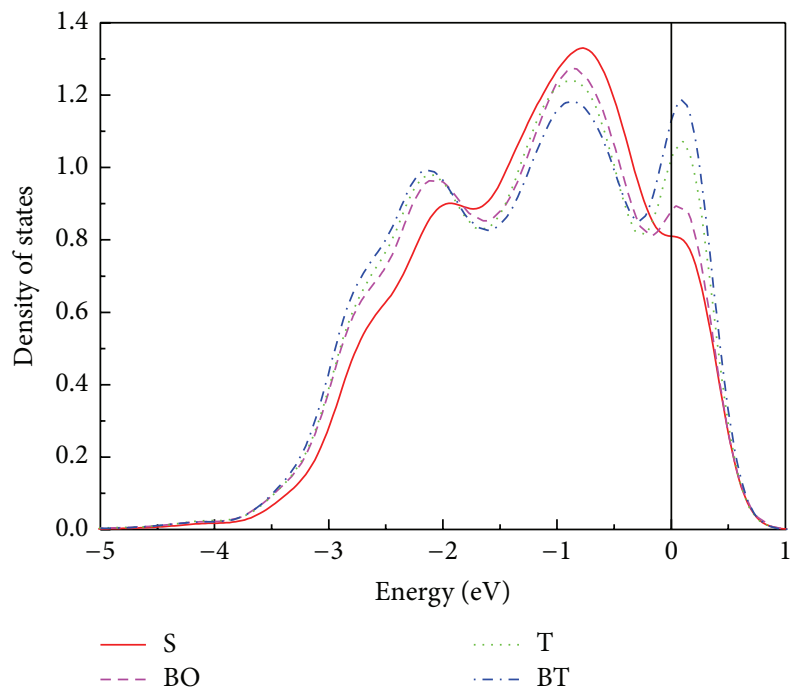

(b)

Figure 3: Local DOS of a He interstitial and its NN-Zr: (a) the s-projected DOS of He and (b) $d$-projected DOS of NN-Zr for the He interstitials at various positions (vertical line is the Fermi level $E_{F}$ ). [37]

Using these values, Young's modulus $E$ can be obtained by

$$
E=\frac{9 B G}{3 B+G}
$$

The elastic anisotropy is expressed by two popular anisotropic indexes, that is, the percentage anisotropy in shear $\left(A_{G}\right)[48]$ and the universal anisotropic index $\left(A^{U}\right)$ [49]. They are defined as

$$
A_{G}=\frac{G_{\mathrm{V}}-G_{\mathrm{R}}}{G_{\mathrm{V}}+G_{\mathrm{R}}}, \quad A^{U}=5 \frac{G_{\mathrm{V}}}{G_{\mathrm{R}}}+\frac{B_{\mathrm{V}}}{B_{\mathrm{R}}}-6 .
$$

These values can range from zero (i.e., completely elastic isotropic) to $100 \%$ (i.e., the maximum anisotropic).

All of the calculated elastic constants results are listed in Table 3. For $\alpha-\mathrm{Zr}$, our calculated compressive moduli $C_{11}$ and $C_{33}$ and shear moduli $C_{12}$ and $C_{13}$ agree well with experimental results (at $298 \mathrm{~K}$ ) showing an accuracy of about $\pm 10 \%$. However, our calculations underestimate the shear modulus $C_{44}$ by about $21 \%$. And these errors seem to also exist in others' reports [31]. There are at least two factors leading to these errors: one is the significance of temperature dependence [38], since first-principle calculations are all conducted at $0 \mathrm{~K}$; the other one is the intrinsic feature of DFT calculations within the ultrasoft pseudo potentials 


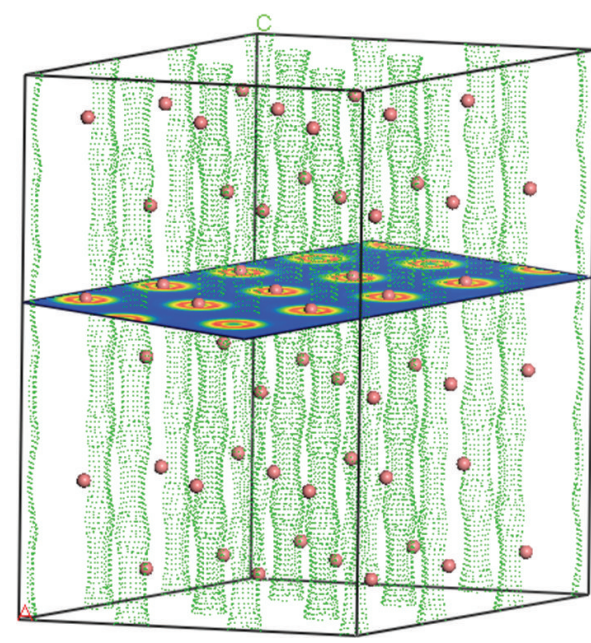

(a)

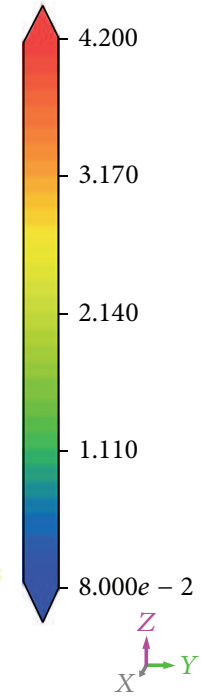

${ }_{X}^{Z} \rightarrow Y$
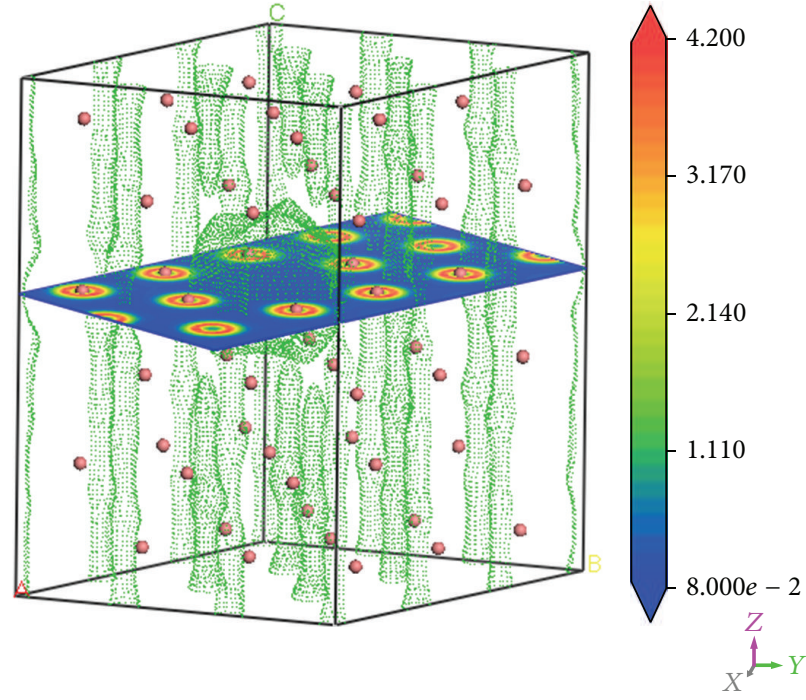

(b)

FIGURE 4: The charge density $(\rho(r))$ in a (a) perfect Zr crystal and (b) 54-atom Zr supercell with one Zr atom substituted by a vacancy. The green dotted surfaces are the isosurfaces of $\rho(r)=140 \mathrm{e} / \mathrm{nm}^{3}$ and the slices are the contour plots of the charge density of the basal planes crossing the $\mathrm{BO}$ and $\mathrm{S}$ sites.

Table 3: Elastic constants $C_{i j}$ (in GPa), bulk modulus $B$ (in GPa), shear modulus $G$ (in GPa), Young's modulus $E$ (in GPa), percentage anisotropy in shear $A_{G}$, and universal anisotropic index $A^{U}$ of $\alpha-\mathrm{Zr}$ and $\mathrm{Zr}$-He systems as calculated in the present work compared with experimental values.

\begin{tabular}{|c|c|c|c|c|c|c|c|c|c|c|}
\hline & $C_{11}$ & $C_{33}$ & $C_{12}$ & $C_{13}$ & $C_{44}$ & $B$ & G & $E$ & $A_{G}$ & $A^{U}$ \\
\hline$\alpha-\mathrm{Zr}$ (this work) & 138.22 & 145.17 & 65.62 & 70.27 & 25.14 & 92.59 & 31.19 & 84.13 & 0.016 & 0.16 \\
\hline$\alpha-\mathrm{Zr}(\mathrm{PBE} / \mathrm{PAW})[36]$ & 157 & 158 & 51 & 62 & 15 & 91 & 29 & 80 & & \\
\hline$\alpha-\mathrm{Zr}$ (PW91/UPP) [31] & 142 & 164 & 64 & 64 & 29 & 92 & & & & \\
\hline$\alpha-\mathrm{Zr}$ (PW91/UPP) [32] & 159.38 & 180.93 & 57.96 & 66.07 & 17.52 & 96.16 & 38.99 & 103.04 & & \\
\hline$\alpha-\mathrm{Zr}(\mathrm{PBE} / \mathrm{UPP})[37]$ & 139.4 & 162.7 & 71.3 & 66.3 & 25.5 & & & & & \\
\hline$\alpha-\operatorname{Zr}$ (Exp.) [38] at $4 \mathrm{~K}$ & 155.4 & 172.5 & 67.2 & 64.6 & 36.3 & & & & & \\
\hline$\alpha-\operatorname{Zr}$ (Exp.) [38] at $298 \mathrm{~K}$ & 143.4 & 164.8 & 72.8 & 65.3 & 32.0 & & & & & \\
\hline $\mathrm{Zr96He}$ (this work) & 139.23 & 145.14 & 64.85 & 69.60 & 24.43 & 92.35 & 31.22 & 84.17 & 0.020 & 0.21 \\
\hline Zr54He (this work) & 144.24 & 147.03 & 60.91 & 69.18 & 23.65 & 92.61 & 32.44 & 87.14 & 0.034 & 0.35 \\
\hline $\mathrm{Zr} 36 \mathrm{He}$ (this work) & 140.99 & 161.41 & 65.62 & 64.74 & 21.98 & 92.46 & 31.47 & 84.80 & 0.045 & 0.47 \\
\hline
\end{tabular}

framework, which is involved in many approximations and simplifications for the sake of calculation time. Despite these errors from experimental values, first-principle methods are still considered to be efficient in calculating elastic constants $[32,37]$.

The differences of elastic constants between diagonal and off-diagonal elements for both $\alpha$ - Zr and $\mathrm{Zr}$-He systems in Table 3 indicate their anisotropy to uniaxial compression and shear, biaxial compression, and distortion [32]. The addition of $\mathrm{He}$ atoms changes elastic constants in an anisotropic way: increasing $C_{11}$ and decreasing $C_{44}$ and $C_{13}$, while changing $C_{12}$ and $C_{33}$ in an irregular way. In particular, $C_{44}$ and $C_{13}$ change linearly with the He concentration. This elastic anisotropy can also be easily seen from the $A_{G}$ and $A^{U}$ values. Both indexes indicate that, with the increasing concentration of $\mathrm{He}, \mathrm{Zr}$ host becomes more elastically anisotropic. Unfortunately, we failed to find any data on the effects of helium concentration on elastic constants in zirconium. Hence, we compared our results with those for hcp-Er [50], of which $C_{11}, C_{33}$, and $C_{44}$ decrease and $C_{12}$ increases linearly with He concentration, while $C_{13}$ changes in an irregular way. This may be due to the crystal distortion caused by interstitial He atoms [50]. The changes of $B, G$, and $E$ values in Table 3 are relatively small compared with those for Er [50]. According to the mechanical stability criteria for hexagonal phase, given by $[51]$

$$
C_{44}>0, \quad C_{11}>\left|C_{12}\right|, \quad\left(C_{11}+2 C_{12}\right) C_{33}>2 C_{13}^{2},
$$

all of the systems we studied are still mechanically stable.

As mentioned above, large concentrations of vacancies can be generated in nuclear structural materials. So we also studied how the interactions between $\mathrm{He}$ and vacancies 


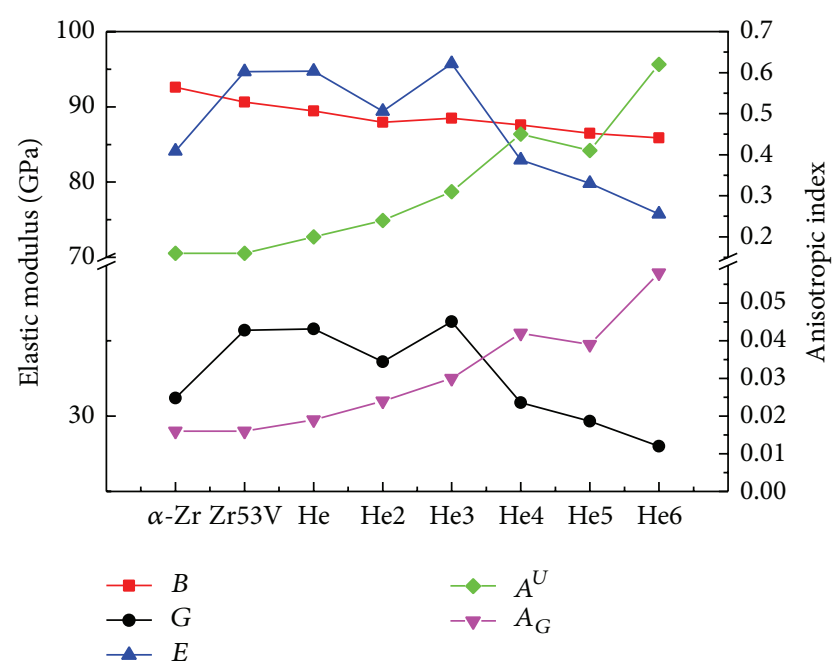

Figure 5: The elastic modulus $(B, G$, and $E$ ) and anisotropic index ( $A^{U}$ and $A_{G}$ ) of $\alpha$-Zr and Zr-V-He systems calculated from Table 4 (He, He2. . represent Zr53VHe, Zr53VHe2. .., resp.).

TABLE 4: Elastic constants $C_{i j}$ (in GPa) of $\alpha$-Zr and Zr-V-He systems.

\begin{tabular}{lccccc}
\hline & $C_{11}$ & $C_{33}$ & $C_{12}$ & $C_{13}$ & $C_{44}$ \\
\hline$\alpha-Z r$ & 138.22 & 145.17 & 65.62 & 70.27 & 25.14 \\
Zr53V & 143.05 & 149.01 & 58.64 & 66.04 & 28.81 \\
Zr53VHe & 143.77 & 145.08 & 56.57 & 64.92 & 28.35 \\
Zr53VHe2 & 140.19 & 143.75 & 59.50 & 62.12 & 25.75 \\
Zr53VHe3 & 144.53 & 153.28 & 55.23 & 61.13 & 26.91 \\
Zr53VHe4 & 136.59 & 148.77 & 59.81 & 61.94 & 21.63 \\
Zr53VHe5 & 133.72 & 144.20 & 61.79 & 60.91 & 21.08 \\
Zr53VHe6 & 130.54 & 151.16 & 62.97 & 58.95 & 18.63 \\
\hline
\end{tabular}

affect the elastic properties. Supercells with $53 \mathrm{Zr}$ atoms, one vacancy, and several He atoms (0 6) were studied. The results are shown in Table 4 and Figure 5. Firstly, when a $\mathrm{Zr}$ atom in a 54-atom supercell is replaced by a vacancy, the elastic anisotropy does not change according to the $A_{G}$ and $A^{U}$ values while the $C_{11}, C_{33}$, and $C_{44}$ values increase and the $C_{12}$ and $C_{13}$ values decrease. The evaluated $B$ value of the polycrystal also decreases a little, in contrast to that of the $G$ and $E$. When more He atoms are added to the vacancy, the elastic anisotropy increases linearly and significantly. At the same time, the elastic moduli $C_{11}, C_{13}$, and $C_{44}$ decrease almost linearly with the increase in the $\mathrm{He}$ concentration, while $C_{33}$ and $C_{12}$ change irregularly. The $B, G$, and $E$ values also decrease linearly with the increase in the He concentration, which is consistent with the case of $\operatorname{Er}$ [50]. Comparing with the single effect of $\mathrm{He}$, the $\mathrm{He}-\mathrm{V}$ complexes have affected the mechanical properties much more obviously. It seems that the presence of $\mathrm{He}-\mathrm{V}$ clusters can lead to a significant deterioration of the mechanical properties of $\mathrm{Zr}$, which may intensively limit their performances. In our future work, we will promote our study by considering the synergistic effects of $\mathrm{He}$ and $\mathrm{H}$ on $\mathrm{Zr}$, since these two elements always cooperate with each other in nuclear environment, producing strengthened effects on nuclear materials.

\section{Conclusion}

In the present study, first-principles calculation has been conducted to investigate the structural stability and electronic and mechanical properties of $\mathrm{Zr}$-He systems. For the perfect $\alpha-\mathrm{Zr}$ crystal, the He BO interstitial site is most energetically favorable, while, for a not perfect one with preexisting vacancies, the $S$ site is most stable. The analysis on the DOS and charge density gives a better understanding about the structural stability issue. It is found that the introduction of He changes the elastic constants in an anisotropic way, leading to a significant increase in elastic anisotropy. And this effect is further enlarged while He-V complexes form, resulting in an intensive deterioration of the mechanical properties of $\mathrm{Zr}$.

\section{Conflict of Interests}

The authors declare that there is no conflict of interests regarding the publication of this paper.

\section{Acknowledgments}

The authors are very grateful for the financial support by the Science Foundation of China Academy of Engineering Physics, China (Grant no. 2010A0301011), and by the National Natural Science Foundation of China (Grant no. 91126001).

\section{References}

[1] T. Schober and K. Farrell, "Helium bubbles in $\alpha$-Ti and Ti tritide arising from tritium decay: a tem study," Journal of Nuclear Materials, vol. 168, no. 1-2, pp. 171-177, 1989.

[2] Y.-L. Wang, S. Liu, L.-J. Rong, and Y.-M. Wang, "Atomistic properties of helium in hcp titanium: a first-principles study," Journal of Nuclear Materials, 2010.

[3] D. Xu, T. Bus, S. C. Glade, and B. D. Wirth, "Thermal helium desorption spectrometry of helium-implanted iron," Journal of Nuclear Materials, vol. 367-370, pp. 483-488, 2007.

[4] S. J. Zenobia, L. M. Garrison, and G. L. Kulcinski, “The response of polycrystalline tungsten to $30 \mathrm{keV}$ helium ion implantation at normal incidence and high temperatures," Journal of Nuclear Materials, vol. 425, no. 1-3, pp. 83-92, 2012.

[5] E. V. Kornelsen and A. A. Van Gorkum, "Study of bubble nucleation in tungsten using thermal desorptionspectrometry: clusters of 2 to 100 helium atoms," Journal of Nuclear Materials, vol. 92, no. 1, pp. 79-88, 1980.

[6] C. S. Becquart and C. Domain, "Migration energy of He in W revisited by Ab initio calculations," Physical Review Letters, vol. 97, no. 19, Article ID 196402, 2006.

[7] R. L. Simons, H. R. Brager, and W. Y. Matsumoto, "Design of a single variable helium effects experiment for irradiation in FFTF using alloys enriched in nickel-59," Journal of Nuclear Materials, vol. 141-143, no. 2, pp. 1057-1060, 1986.

[8] T. Ishizaki, Q. Xu, T. Yoshiie, S. Nagata, and T. Troev, “The effect of hydrogen and helium on microvoid formation in iron and 
nickel," Journal of Nuclear Materials, vol. 307-311, no. 2, pp. 961965, 2002.

[9] J. D. Hunn, E. H. Lee, T. S. Byun, and L. K. Mansur, "Helium and hydrogen induced hardening in 316LN stainless steel," Journal of Nuclear Materials, vol. 282, no. 2-3, pp. 131-136, 2000.

[10] N. Sekimura, T. Iwai, Y. Arai et al., "Synergistic effects of hydrogen and helium on microstructural evolution in vanadium alloys by triple ion beam irradiation," Journal of Nuclear Materials, vol. 283-287, pp. 224-228, 2000.

[11] T. Schober, H. Trinkaus, and R. Lässer, "A TEM study of the aging of Zr tritides," Journal of Nuclear Materials, vol. 141-143, no. 1, pp. 453-457, 1986.

[12] T. Schober and R. Lässer, “The aging of zirconium tritides: a transmission electron microscopy study," Journal of Nuclear Materials, vol. 120, no. 2-3, pp. 137-142, 1984.

[13] T. Hayashi, T. Suzuki, and K. Okuno, "Long-term measurement of helium-3 release behavior from zirconium-cobalt tritide," Journal of Nuclear Materials, vol. 212-215, pp. 1431-1435, 1994.

[14] B. Cox, "Pellet-clad interaction (PCI) failures of zirconium alloy fuel cladding-a review," Journal of Nuclear Materials, vol. 172, no. 3, pp. 249-292, 1990.

[15] B. A. Cheadle, C. E. Ells, and W. Evans, "The development of texture in zirconium alloy tubes," Journal of Nuclear Materials, vol. 23, no. 2, pp. 199-208, 1967.

[16] A. Yilmazbayhan, A. T. Motta, R. J. Comstock, G. P. Sabol, B. Lai, and Z. Cai, "Structure of zirconium alloy oxides formed in pure water studied with synchrotron radiation and optical microscopy: relation to corrosion rate," Journal of Nuclear Materials, vol. 324, no. 1, pp. 6-22, 2004.

[17] R. N. Singh, A. K. Bind, N. S. Srinivasan et al., "Influence of hydrogen content on fracture toughness of CWSR Zr-2.5 Nb pressure tube alloy," Journal of Nuclear Materials, vol. 432, no. 1, pp. 87-93, 2012.

[18] S. C. Lumley, S. T. Murphy, P. A. Burr et al., "The stability of alloying additions in Zirconium," Journal of Nuclear Materials, vol. 437, no. 1, pp. 122-129, 2013.

[19] C. B. A. Forty and P. J. Karditsas, "Uses of zirconium alloys in fusion applications," Journal of Nuclear Materials, vol. 283-287, pp. 607-610, 2000.

[20] A. A. Lucas, "Helium in metals," Physica B: Physics of Condensed Matter, vol. 127, no. 1-3, pp. 225-239, 1984.

[21] Y. Dai, G. R. Odette, and T. Yamamoto, “The effects of helium in irradiated structural alloys," in Comprehensive Nuclear Materials, pp. 141-193, Elsevier, 2012.

[22] D. K. Sood, M. Sundararaman, S. K. Deb, R. Krishnan, and M. K. Mehta, "Blistering of zirconium, inconel-718 and stainless steel316 by $2 \mathrm{MeV}$ helium ions," Journal of Nuclear Materials, vol. 79, no. 2, pp. 423-425, 1979.

[23] R. H. Zee, J. F. Watters, and O. M. Westcott, "On the critical dose for blistering in helium irradiated zirconium and $\mathrm{ZrNb}$," Journal of Nuclear Materials, vol. 115, no. 1, pp. 131-133, 1983.

[24] H. Zheng, S. Liu, H. B. Yu, L. B. Wang, C. Z. Liu, and L. Q. Shi, "Introduction of helium into metals by magnetron sputtering deposition method," Materials Letters, vol. 59, no. 8-9, pp. 10711075, 2005.

[25] Y. M. Koroteev, O. V. Lopatina, and I. P. Chernov, "Structure stability and electronic properties of the $\mathrm{Zr}$-He system: firstprinciples calculations," Physics of the Solid State, vol. 51, no. 8, pp. 1600-1607, 2009.

[26] Q. Peng, W. Ji, H. Huang et al., "Stability of self-interstitial atoms in hcp-Zr," Journal of Nuclear Materials, vol. 429, no. 1, pp. 233236, 2012.
[27] Q. Peng, W. Ji, H. Huang et al., "Axial ratio dependence of the stability of self-interstitials in HCP structures," Journal of Nuclear Materials, vol. 437, no. 1, pp. 293-296, 2013.

[28] G. D. Samolyuk, S. I. Golubov, Y. N. Osetsky et al., "Selfinterstitial configurations in hcp Zr: a first principles analysis," Philosophical Magazine Letters, vol. 93, no. 2, pp. 93-100, 2013.

[29] G. Vérité, C. Domain, C. C. Fu et al., "Self-interstitial defects in hexagonal close packed metals revisited: evidence for lowsymmetry configurations in Ti, Zr, and Hf," Physical Review B, vol. 87, no. 13, Article ID 134108, 2013.

[30] F. Wang and H. R. Gong, "First principles study of various Zr$\mathrm{H}$ phases with low $\mathrm{H}$ concentrations," International Journal of Hydrogen Energy, vol. 37, no. 17, pp. 12393-12401, 2012.

[31] C. Domain, R. Besson, and A. Legris, "Atomic-scale Ab-initio study of the Zr-H system: I. Bulk properties," Acta Materialia, vol. 50, no. 13, pp. 3513-3526, 2002.

[32] W. Zhu, R. Wang, G. Shu, P. Wu, and H. Xiao, "First-principles study of different polymorphs of crystalline zirconium hydride," Journal of Physical Chemistry C, vol. 114, no. 50, pp. 2236122368, 2010.

[33] S. K. Sikka, Y. K. Vohra, and R. Chidambaram, "Omega phase in materials," Progress in Materials Science, vol. 27, no. 3-4, pp. 245-310, 1982.

[34] L. Yang, S. M. Peng, X. G. Long et al., "Ab initio study of intrinsic, $\mathrm{H}$, and He point defects in hcp-Er," Journal of Applied Physics, vol. 107, no. 5, Article ID 054903, 2010.

[35] L. Yang, S. M. Peng, X. G. Long et al., "Ab initio study of stability and migration of $\mathrm{H}$ and He in hcp-Sc," Journal of Physics Condensed Matter, vol. 23, no. 3, Article ID 035701, 2011.

[36] J. Blomqvist, J. Olofsson, A. M. Alvarez et al., "Structure and thermodynamical Properties of Zirconium hydrides from firstprinciple," in Proceedings of the 15th International Conference on Environmental Degradation of Materials in Nuclear Power Systems-Water Reactors, 2012.

[37] H. Ikehata, N. Nagasako, T. Furuta, A. Fukumoto, K. Miwa, and T. Saito, "First-principles calculations for development of low elastic modulus Ti alloys," Physical Review B-Condensed Matter and Materials Physics, vol. 70, no. 17, Article ID 174113, 2004.

[38] E. S. Fisher and C. J. Renken, "Single-crystal elastic moduli and the hcp $\rightarrow$ bcc transformation in Ti, Zr, and Hf," Physical Review, vol. 135, no. 2A, article A482, 1964.

[39] S. J. Clark, M. D. Segall, C. J. Pickard et al., "First principles methods using CASTEP," Zeitschrift fur Kristallographie, vol. 220, no. 5-6, pp. 567-570, 2005.

[40] D. Vanderbilt, "Soft self-consistent pseudopotentials in a generalized eigenvalue formalism," Physical Review B, vol. 41, no. 11, article 7892, 1990.

[41] J. P. Perdew, K. Burke, and M. Ernzerhof, "Generalized gradient approximation made simple," Physical Review Letters, vol. 77, no. 18 , article $3865,1996$.

[42] B. G. Pfrommer, M. Côté, S. G. Louie, and M. L. Cohen, "Relaxation of crystals with the Quasi-Newton method," Journal of Computational Physics, vol. 131, no. 1, pp. 233-240, 1997.

[43] X. T. Zu, L. Yang, F. Gao et al., "Properties of helium defects in bcc and fcc metals investigated with density functional theory," Physical Review B-Condensed Matter and Materials Physics, vol. 80, no. 5, Article ID 054104, 2009.

[44] R. Pentcheva and M. Scheffler, "Initial adsorption of Co on $\mathrm{Cu}(001)$ : a first-principles investigation," Physical Review BCondensed Matter and Materials Physics, vol. 65, no. 15, Article ID 155418, 2002. 
[45] W. Voigt, Lehrbuch der Kristallphysik, Teubner, Leipzig, Germany, 1928.

[46] A. Reuss and Z. Angnew, "A calculation of the bulk modulus of polycrystalline materials," Mathematical Methods, vol. 9, article 55, 1929.

[47] R. Hill, "The elastic behaviour of a crystalline aggregate," Proceedings of the Physical Society A, vol. 65, no. 5, article 349, 1952.

[48] D. H. Chung, W. R. Buessem, F. W. Vahldiek et al., Anisotropy in Single Crystal Refractory Compounds, Plenum Press, New York, NY, USA, 1968.

[49] S. I. Ranganathan and M. Ostoja-Starzewski, "Universal elastic anisotropy index," Physical Review Letters, vol. 101, no. 5, Article ID 055504, 2008.

[50] F. Kai-Min, Y. Li, S. Qing-Qiang et al., "First-principles study on elastic properties of hexagonal phase $\operatorname{ErAx}(\mathrm{A}=\mathrm{H}, \mathrm{He})$, , Acta Physica Sinica, vol. 62, no. 11, Article ID 116201, 2013.

[51] Z.-J. Wu, E.-J. Zhao, H.-P. Xiang, X.-F. Hao, X.-J. Liu, and J. Meng, "Crystal structures and elastic properties of superhard Ir $\mathrm{N}_{2}$ and $\mathrm{Ir} \mathrm{N}_{3}$ from first principles," Physical Review BCondensed Matter and Materials Physics, vol. 76, no. 5, Article ID 054115, 2007. 

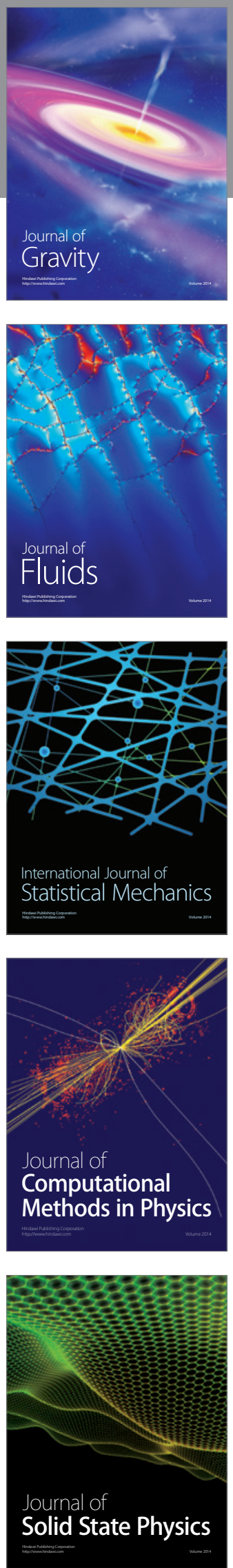

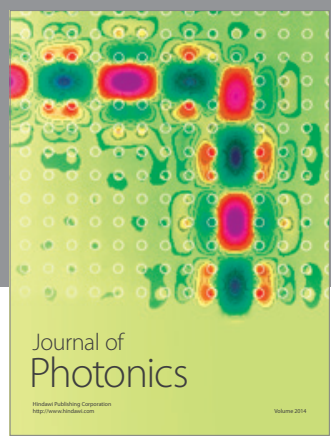

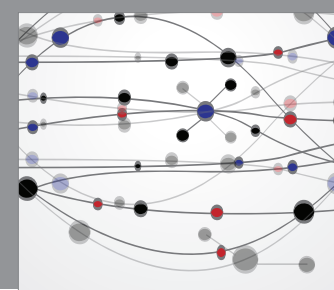

The Scientific World Journal

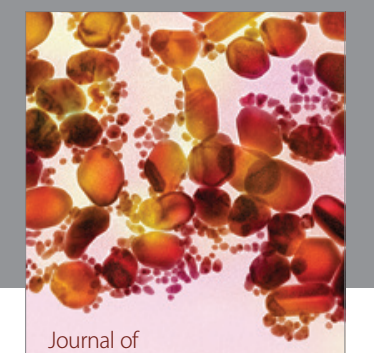

Soft Matter
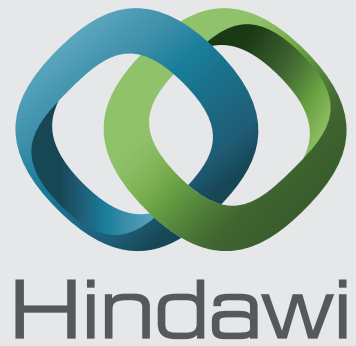

Submit your manuscripts at

http://www.hindawi.com
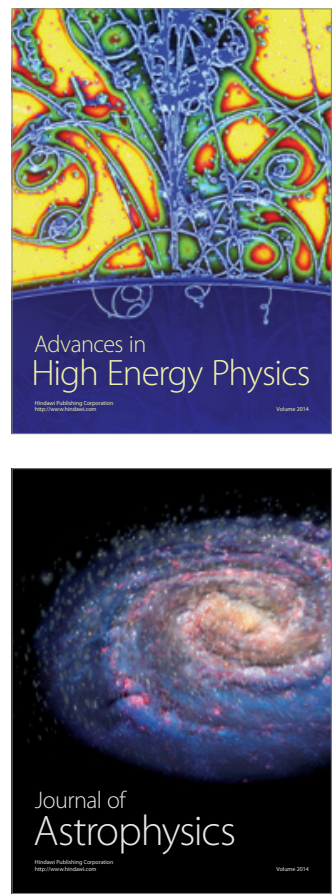
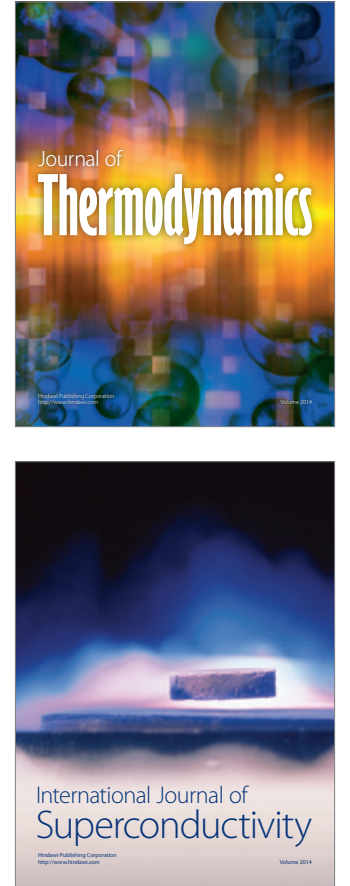
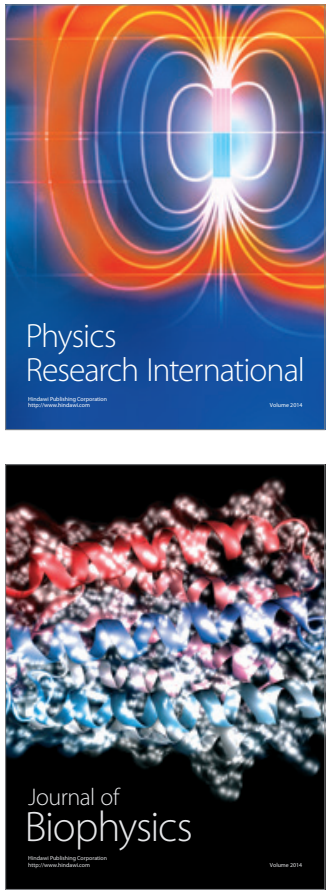
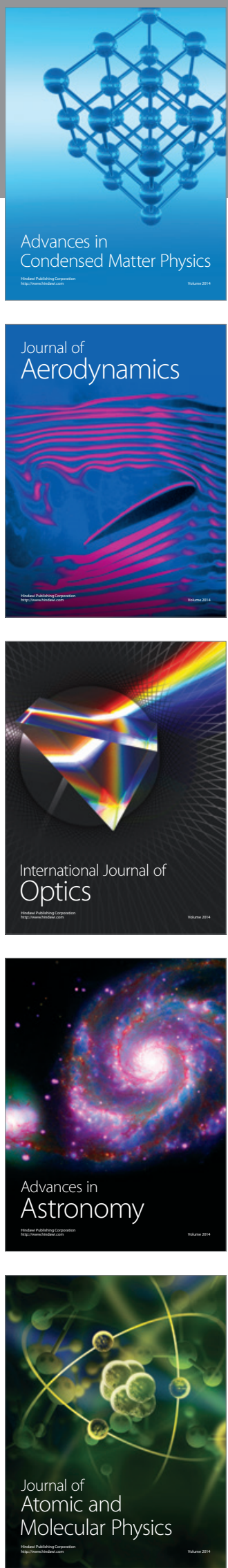